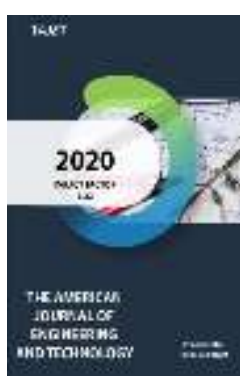

\title{
The General Structure Of The Microprocessor
}

\author{
Oybek Bakhtiyorjon ogli Parpiev \\ Assistant, “EEE” Chair, Andijan Machine-Building Institute, Uzbekistan
}

\begin{abstract}
Journal Website: http://usajournalshub.c om/index,php/tajet

Copyright: Original content from this work may be used under the terms of the creative commons attributes 4.0 licence.
\end{abstract}

\section{ABSTRACT}

Synchronous motors used by the technological process in the production of industrial enterprises are considered to be the organ of mechanical drives of pipe compressors. When high-voltage, high-power motors are excited by the automatic control method, the setting nominal excitation current is maintained, and when such equipment is operated, a high energy saving is achieved.

Such problems are the problems of the quarry and the demand for production. When solving these problems, the replacement of inefficient tristor excitatory devices with effective microprocessor exciters is set forth in the report of the master's thesis.

\section{KEYWORDS}

Tiristor, mikroprosessor, energy, resources savings, capacitance energy, stirrer-up.

\section{INTRODUCTION}

The core of any microprocessor system is the microprocessor or processor. In other words, a "processor" is a microprocessor that is a node that processes all data within a microprocessor system. The rest of the nodes perform auxiliary functions: data storage, communication with external devices, communication with the user. The microprocessor performs arithmetic and logic operations, temporary storage of codes, exchange of information between nodes of microprocessor systems and other functions. The processor is comparable to a system brain. 


\section{MATERIALS AND METHODS}

The processor performs a series of operations. We know that a microprocessor can perform operations in parallel. The advantage of sequential data processing is that complex operations can be performed in a single clock process. But the execution of operations depends on their ease or complexity. As a result, the microprocessor performs any operations, but all operations are performed on a single node. (Figure 1).

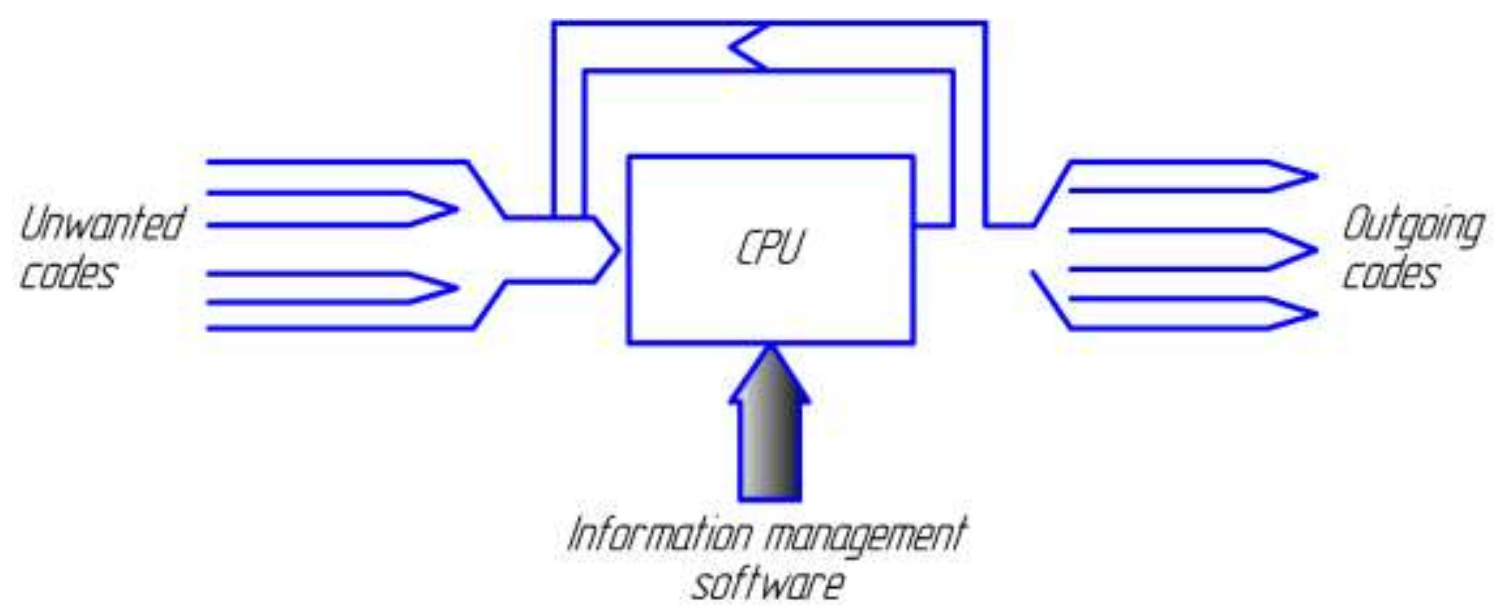

Figure 1. Information flow in microprocessors.

The basis of the processor is the command system. The command system and structure determine the speed, flexibility, and ease of use of the processor.

Microprocessor commands can range from tens to hundreds. Command codes come in a variety of bit lengths. Each command has its own execution time, so the execution time of a program depends not only on the program, but also on what commands are executed. The processor structure includes: registers, arithmetic logic devices, multiplexers, buffers, and registers. The work process of all nodes is carried out by a common external leaving signal of the processor. The processor is a complex digital device. (Figure 2). 


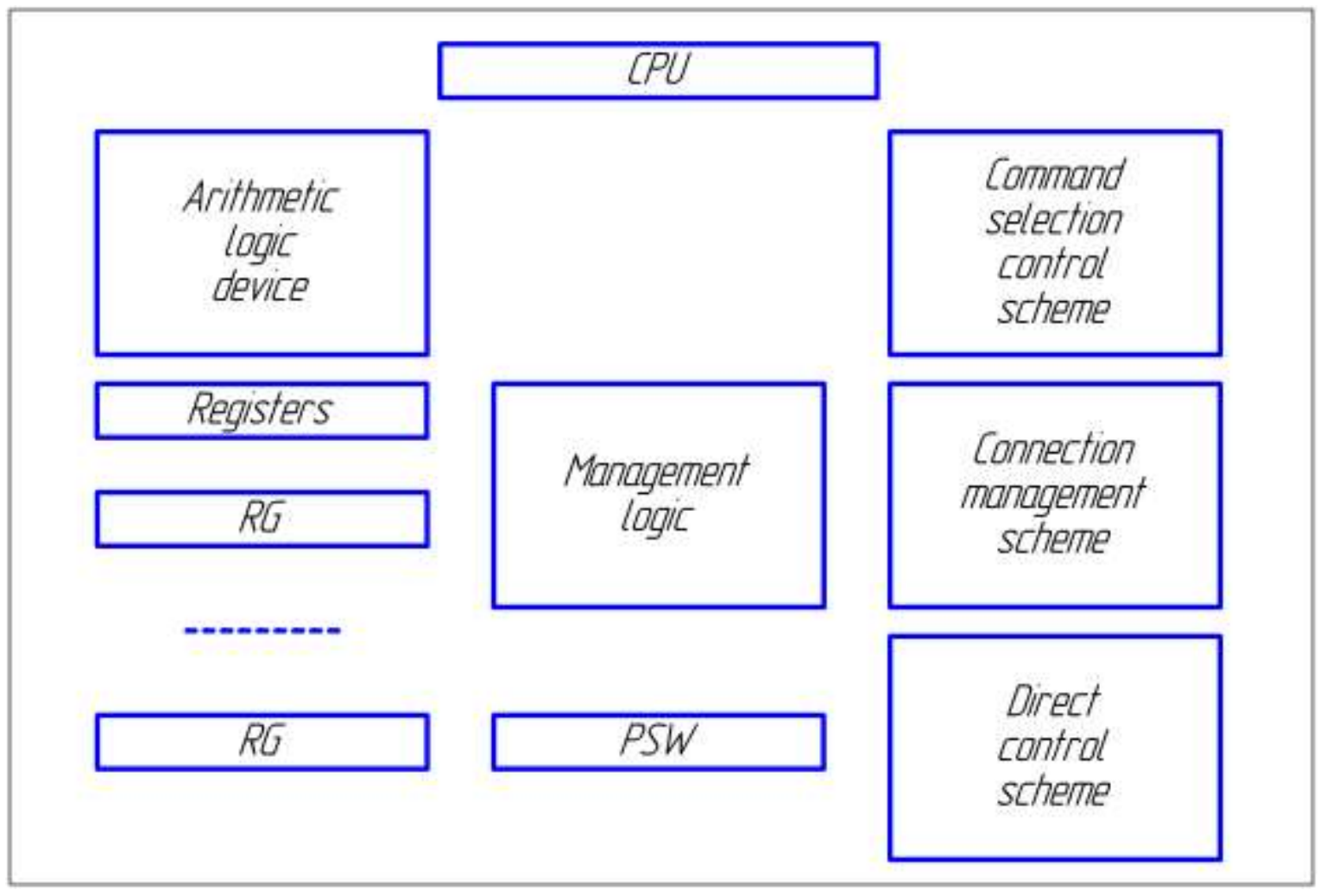

Figure 2. The structure of a simple microprocessor.

The command selection control circuit reads and decrypts the commands.

The first microprocessors did not have the ability to read the previous command and select the next command at the same time, because the processor did not include these operations. However, today's 16-bit processors have this feature, which includes a command called a conveyor, which allows you to select and execute subsequent commands during the execution of one instruction. This speeds up the work process. The conveyor contains three small processor memories (with the release of the external bus), which means that a few commands can be written to this memory in a short time. These commands are read from the processor, like a conveyor, in their own state. However, the executed command goes to the memory cell, rejecting many remaining (queued) commands in memory.

As the conveyor improved, more cache was added. In this way, the processor contains the commands that need to be executed during the execution of the commands. The larger the cache, the more the processor is able to store commands in the next pass.

The cache stores commands that the processor must execute at the moment. For faster processing of commands, modern processors use a selection and decoding complex, parallel commands conveyor mode. This allows you to know the transition period and other methods of commands.

Arithmetic is a logical device. This device performs the same functions as processing 
data when executing processor commands. Examples of processing include logical operations (for example, logical "AND", "OR"), as well as bit operations on operands and arithmetic operations (multiplication, division, etc.). The type of command being executed includes the codes with which the operation is performed and their results.

Let's take a look at the sequence of data processing - arithmetic or logical functions. In most cases, this works with a pair of operands, that is, the executing operand destinaion and the operand source src (source). The usual scheme of operation of this instruction is as follows: dest $=F$ (dest, serc), where $F$ is a function of two variables. This converts the processor to a binary number from the above when the instruction (register, memory, constant instruction) is executed, and records the results on one of the destinations. You will need another even number for the same function. The speed of the AMQ (arithmetic logic device) indicates the performance of the processor. Not only is the clock frequency of the AMQ important, but the number of beats is also important for the execution of this or that command. In order to increase efficiency, manufacturers equalize the execution time of the command by one clock, thus ensuring the high frequency operation of the AMQ. One way to do this is to reduce the number of instructions that can be executed in the AMQ and the number of commands that must be present in the processor (RISC - processors). Another way is for AMQs to execute commands at the same time.

For special complex variable operations, processor systems were programmed with simple commands and special internal programs, but later special computing parts were created. Mathematical coprocessors, that is, can be replaced over time by these coprocessors. In modern microprocessors, mathematical coprocessors are part of the microprocessor.

Processor registers are used to store various codes, such as data, addresses, and working codes, for cache and temporary storage. The operation performed by these codes is significantly faster on the processor, in general, the presence of such regulators in the processor structure leads to positive results. A high-speed processor is inextricably linked to the register bit. Discharge registers in AMQ may not be compatible with external discharges.

Internal registers are divided into two types according to the execution function. The first is Intel's registers, which type of registry incorporates a clear structural response. On the one hand, such features make it easier for businesses to run these types of registers, and reduce the time it takes to execute orders. On the other hand, these registers reduce the stability of the processor and slow down the performance of the program. For example, some arithmetic input and output operations on devices are performed through a single register - the accumulator, resulting in a jump between registers to perform certain processes. The second type is that all (almost all) registers perform the same functions, that is, DES T-11 16-bit processors. In this way, high stability is achieved, complicating the processor circuit. There are also intermediate processor types. Half of the registers available on this type of Motorola's MS6800 processor are used for data, but they are interchangeable.

Status (flag) register is also important, but it is also a component of the processor. This register does not contain information about data or addresses. It includes the word processor status (PSX). Each bit in this word 
(flag) contains information about the result of the executed command. For example, there is a bit of the result zero, and when this result is executed, the result of the command is zero. These bits (flags) are executed in conditional transitions. Also, these registers sometimes contain control commands, which determine the mode of execution of some commands.

Interrupt management schemes are interrupt requests received by the processor that determine the starting address of interruptions in the program. PSW (Processor Status Word) is the word for processor status. For example, if we assume that there is a zero result state, then the result of the previous executed command is zero, or if the state differs from zero, then it is erased from the processor memory. These bits are used to pass commands conditionally. For example, commands only go into hibernation when there is a zero result. This registry sometimes has control flags to determine the mode of certain commands.

A direct control circuit is a circuit that allows the memory to be temporarily disconnected from the processor's external bus and the processor to be temporarily accessed directly from the device.

Control logic - interacts with all nodes of the processor, transmits data, synchronizes the processor with external signals and responds to the input and output of information. This is technically the "hard logic" of the microprocessor.

In this case, the processor's command selection scheme is sequentially retrieved from memory, then the commands are executed, and if necessary, the AMQ is used to process the data. The AMQ input can transmit processed data from memory or internal registers. The internal registry memory can store address codes that need to be processed. The data processing data in the AMQ changes the state of the status 41 register and writes it to the internal memory. If necessary, the data can be overwritten from memory, from the internal register.

However, microprocessor systems do not matter to the programmer. The programmer should pay attention to the processor as a "black box". This allows you to convert incoming and outgoing codes to outgoing codes. The programmer is required to know the command system, the operating mode of the processor, and the interaction of the processor with external devices. As for the internal state of the processor system, it can be done if the processor knows that the command state or modes work in one or another case.

A microprocessor (MP), otherwise known as a central processing unit (CPU), is a softwarecontrolled, functionally complete device that processes information and is designed to accommodate one or more large (KIS) or very large (JKIS) integrated circuits.

The microprocessor performs the following functions:

- Read and decrypt commands from main memory (AX);

- Read data from $A X$ and registers of external device (TK) adapters;

- Receiving and processing requests and commands from adapters for maintenance of TQs;

- Data processing and recording them in the registers of $A X$ and TQ, adapters;

- Development of control signals for all other nodes and blocks of the computer.

The discharge of the microprocessor data bus determines the discharge of the PC; The 
resolution of the MP address bus determines its address width.

The discharge of the microprocessor data bus determines the discharge of the PC; The resolution of the MP address bus determines its address width.

The address width is the maximum number of main memory cells that can be addressed directly by the microprocessor.

\section{CONCLUSION}

So, in order to achieve this goal, it was decided to solve the following task:

Use of thyristor microprocessors in the control of synchronous and asynchronous motors, if possible, depending on the conditions of production processes;

The general structure of the microprocessor is considered in order to consider the problem in detail.

\section{REFERENCES}
1. X.Y.Abasxanova., U.B.Amirsaidov. "Microprocessors". Tashkent "Fan va texnologiya" publishing house - 2016.
2. A.Raximov, N.Raximov, M.Mamadaliev, N.Sadritdinov, M.Najmiddinova. "Practicum in electrical engineering and radio engineering". Tashkent - „O'qituvchi" publishing house - 1983.
3. X.F.Fozilov. "Primary Electrical Engineering". Uzbek State Publishing House Tashkent — 1949.

"In the control of electric drive devices study of microprocessor thyristor drives". Ministry of higher and secondary specialized education of the Republic of Uzbekistan scientific and technical journal of Namangan institute of engineering and technology. ISSN 2181-8622. 187193.

5. Alijanov Donyorbek Dilshodovich*, Boltaboyev Isroiljon Maxammatismoilovich** PhD, Andijan Machine-building institute, Uzbekistan. Photosensitive Sensors in Automated Systems. International Journal of Research Studies in Electrical and Electronics Engineering (IJRSEEE). Volume 6, Issue 2, 2020, PP 33-34. ISSN 2454-9436 (Online).

6. Donyorbek Dilshodovich Alijanov1, Nematjon Rakhimovich Rakhimov2. 1Andijan Machine-Building Institute, Andijan, Uzbekistan.2Ufa State Oil Technical University, Ufa, Russia. Optoelectronic Method for Determining the Physicochemical Composition of Liquids. (c) Automation and software engineering. 2020, №2 (32).

7. Alijanov D.D. Optocoupler based on APV - receiver. Descendants of Muhammad al-Khwarizmi, № 3 (13), September 2020. 